\title{
Resonant transmission through finite-sized carbon nanotubes
}

\author{
Daniel Orlikowski, ${ }^{1}$ Hatem Mehrez, ${ }^{2}$ Jeremy Taylor, ${ }^{2}$, Hong Guo, ${ }^{2}$ Jian Wang, ${ }^{3}$ and Christopher Roland ${ }^{1}$ \\ ${ }^{1}$ Department of Physics, The North Carolina State University, Raleigh, North Carolina 27695 \\ ${ }^{2}$ Center for the Physics of Materials and Department of Physics, McGill University, Montreal, PQ Canada H3A 2T8 \\ ${ }^{3}$ Department of Physics, The University of Hong Kong, Pokfulam Road, Hong Kong, China
}

(Received 26 October 2000; published 28 March 2001)

\begin{abstract}
We have investigated theoretically the conductance through finite-sized carbon nanotubes coupled to featureless leads in the context of standard tight-binding models. Conduction takes place via resonant tunneling, and the resultant spectrum of peaks may be understood in terms of the band structure of the nanotubes. Specific nanotubes display both on- and off-resonance behavior as a function of nanotube length depending upon whether or not the bands cross at a nonzero $k$ value. We have also investigated the approach to the infinite limit in detail, and, in general, find that the finite-size effects can persist out to hundreds of nanometers. Since the manipulation of nanotubes into devices is likely to induce defects, we have investigated their effects on the conduction. The effects of bending and two broad classes of defects, i.e., defect in the absence and/or presence of addimers, were considered. In general, the presence of defects leads both to a reduction and shifting of the resonant peaks at the Fermi level. However, in the infinite limit, low concentrations of defects have only a relatively modest effect on the transport properties. Finally, we have investigated the effects of an externally imposed magnetic field oriented perpendicular to the nanotube axis. The magnetic field shifts the levels, thereby turning on- and off-resonant devices into each other. All of the effects discussed here are testable experimentally.
\end{abstract}

DOI: $10.1103 /$ PhysRevB.63.155412

PACS number(s): 61.48.+c, 72.80.Rj, 73.23.Ad, 73.61.Wp

\section{INTRODUCTION}

There is currently intense interest in the electronic properties of carbon nanotubes. ${ }^{1}$ Carbon nanotubes are either metals or semiconductors depending on their helicity [which is denoted by a pair of integers $(n, m)]$, and therefore have the capability of forming an all carbon nanotube-based molectronic system. To explore this exciting possibility, there has been a considerable amount of experimental ${ }^{2-18}$ and theoretical ${ }^{19-34}$ work. Progress in this field has been rapid, and already several prototypical devices have been constructed and demonstrated with the aid of nanomanipulators. ${ }^{3-8}$ Recently, this field of research has entered a new phase with the formation of hybrid device structures, in which the nanotubes are contacted electronically to other materials. This is a crucial step, as a nanotube-based electronics is only possible when the tubes are efficiently coupled to external leads. Examples of such devices include carbon nanotube magnetic tunnel junctions, ${ }^{16,29}$ nanotube heterojunctions, ${ }^{17}$ and different superconducting junctions. ${ }^{18,31}$ These systems are interesting not only because of their high potential for technolgical applications, but also from a fundamental point of view as a suitable laboratory for physics at the nanometer length scale.

Experimentally and theoretically, there is now considerable evidence that transport through finite-sized carbon nanotube devices, as shown schematically in Fig. 1, takes place via resonant transmission. ${ }^{12-14}$ However, most of the theoretical analysis to date is based on transmission through "infinite" carbon nanotubes, whose character is fundamentally different from that of tubes of finite length coupled to different leads. ${ }^{2,20}$ To fill this gap, we have carried out a largescale theoretical investigation of such systems in which the nanotubes are modeled with standard tight-binding Hamilto- nians coupled to featureless leads, with a focus on the general and universal aspects of the transmission. For the most part, these depend only on the dimensions and symmetries of the carbon nanotubes. Specifically, we have investigated the transport characteristics through pristine and defective nanotubes of different lengths and helicities, along with the effects of an externally imposed magnetic field oriented perpendicular to the nanotube axis. With simple models, it is possible to treat theoretically systems that actually approach the experimental sizes, and thereby elucidate the "finitesize" characteristics of nanotube devices which, in agreement with the experimental results, can actually persist over length scales of hundreds of nanometers.

Considering quantum transport through nanotubes coupled to leads, we have previously shown that the conductance is length dependent. ${ }^{29}$ In particular, for armchair nanotubes of length $L=3 N+1$, with integer $\mathrm{N}$ denoting the number of carbon repeat units, the nanotubes have a large conductance due to a crossing of the scattering states at the Fermi energy. Nanotubes whose lengths are incommensurate with $3 N+1$ give rise to much smaller conductances due to a

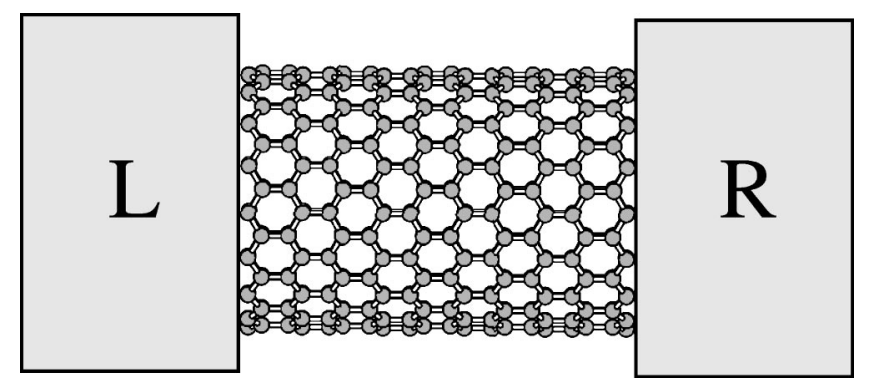

FIG. 1. Schematic of typical device consisting of a nanotube coupled to a left (L) and right (R) lead. 
gap between the scattering states. We shall refer to such systems as on- and off-resonant devices, respectively. Experimentally, such energy gaps, along with conductance oscillations on finite nanotubes, have already been detected with different scanning probes. ${ }^{12-14}$ In this paper, we extend our previous theoretical work ${ }^{29}$ on armchair nanotubes with an investigation of the distribution of the resonant tunneling peaks for nanotubes with different helicities, diameters, and lengths. In general, as the tubes become longer, the number of resonant peaks proliferates and the spacing between the peaks decreases. At the same time, the peak width narrows. Ultimately, when coupled to the leads which serve to broaden the linewidths, the peaks begin to overlap and infinite nanotube results are recovered. Qualitatively similar results are obtained for nanotubes with a different helicity, with the characteristic spectrum of each of the type of nanotube intimately reflecting its band structure.

An important characteristic of quantum transport is shot noise. Shot noise, which derives from the quantization of electric charge, has been the subject of considerable investigations in the field of mesoscopic physics. ${ }^{35-44}$ With shot noise one is able to probe the kinetics of the electrons ${ }^{43}$ and the correlations of the electronic wave functions. ${ }^{44}$ Classical shot noise is associated with an uncorrelated current pulse as electrons cross a device, and gives rise to Poissonian statistics such that the current fluctuations over a given range of frequencies $\Delta \omega$ is proportional to the current: i.e., $<(\Delta I)^{2}$ $>=\left(2 e^{2} / h\right) \Delta \omega I$. In general, both the Pauli Exclusion Principle and Coulombic interactions act to reduce the shot noise from its classical value. For instance, the Coulomb interaction keeps nearby electrons apart and more regularly spaced, so that the number of electrons crossing a given device is less than truly random. In general, these factors act to supress shot noise. However, in the case of negative differential resistance, the shot noise is actually enhanced. We have calculated the shot noise for carbon nanotubes in the regime of noninteracting electron regime for both on- and off-resonant devices. In general, we find that the classical value is suppressed by a factor of about $1 / 4$.

An understanding of the behavior of nanotube devices involves not only the investigation of pristine but also of defective carbon nanotubes. This is important, because the process of positioning the nanotube involves substantial manipulation with either scanning tunneling microscopy (STM) and atomic force microscopy (AFM) tips, which is likely to introduce a defects into the system. We have therefore investigated the properties of two classes of defects associated with strained carbon nanotube, plus finite-sized, bent tubes. The first class of defects is associated with the rotation of a bond on the nanotube wall, i.e., a Stone-Wales rotation, ${ }^{45}$ which yields a (5-7-7-5) defect. Depending on the helicity, temperature, and conditions of strain, this defect undergoes either ductile or brittle behavior, and thereby dominates the mechanical response of pristine nanotubes. ${ }^{46}$ The second class of defects is associated with the presence of addimers on strained carbon nanotubes. Such addimers are likely to be present on as-grown nanotubes, or they may be placed there by direct deposition or with an STM/AFM tip. On strained nanotubes, the addimers give rise to extended defects that may ultimately evolve into short segments of a nanotube with an altered helicity. ${ }^{28}$ In general, we find that the response of the defective nanotube varies with both the helicity and diameter. In some cases, there are significant shifts and reduction in the peaks, so that for a given bias voltage, an effective metal-semiconductor transition is induced. Similar results are obtained for the case of an externally imposed magnetic field that is oriented perpendicular to the nanotubes. In this case, the magnetic field can change on/off resonant devices into each other, and thereby effectively inducing a metal/semiconductor transition.

An outline of this paper is as follows. In the next section, we briefly review the Green's function methodology used to study the conductance of carbon nanotubes. Section III presents the results of our study, with a focus on the statistics of the resonant tunneling peaks, the effects of defects, and that of a magnetic field that is oriented perpendicular to the nanotube axis. Section IV is reserved for a short summary of our results.

\section{METHODOLOGY}

In this section, we briefly outline the methodology used to calculate the conductance of the different carbon nanotube devices. As the theoretical techniques used to define these quantities are well known and outlined extensively elsewhere, ${ }^{19,26,27,47}$ our description will be brief and restricted to issues directly effecting the calculations.

The conductance $G$ between two leads is defined in terms of the current $I$ and voltage $V$ through the relation $I=G V$. The dc conductance is then given by the Landauer formula $G=\left(2 e^{2} / h\right) \operatorname{Tr}(T),{ }^{47}$ where $T$ is the transmission function given by

$$
T=\left(\Gamma_{L} G_{o}^{r} \Gamma_{R} G_{o}^{a}\right),
$$

where $G_{o}^{r, a}$ represent the retarded and advanced Green's function of the nanotube, and $\Gamma_{L, R}$ the couplings of the nanotube to the left $(\mathrm{L})$ and right $(\mathrm{R})$ leads, respectively. The Green's function $\left(G_{o}\right)$ of the nanotube as a function of the Fermi energy $E$ is defined via

$$
G_{o}=\frac{1}{E-H_{\text {tube }}-\Sigma^{r}}
$$

with $\Sigma^{r}=\Sigma_{L}^{r}\left(E-e V_{L}\right)+\Sigma_{R}^{r}\left(E-e V_{R}\right)$ representing the selfenergy terms due to the leads and $H_{\text {tube }}$ the Hamiltonian of the nanotubes. The self-energy terms also give the couplings $\Gamma$ through the relation

$$
\Gamma_{L, R}=i\left(\Sigma_{L, R}^{r}-\Sigma_{L, R}^{a}\right),
$$

so that all of the interactions between the nanotubes and the leads are defined in terms of the self-energy $\Sigma_{L, R}^{r}$ terms. We couple the nanotubes to featureless leads, so that these terms are parametrized by the corresponding line-width functions $\Gamma_{L, R}=-2 \operatorname{Im}\left(\sum_{L, R}^{r}\right)$. For the case of "infinite" nanotubes, 1.e., a nanotube coupled seemlessly to two nanotube leads of the same type, the self-energy terms were calculated via a surface Green's function matching technique. ${ }^{26}$ The nano- 
tube Hamiltonian $H_{\text {tube }}$ was calculated using two different tight-binding models: a nearest neighbor, single-band $\pi$-orbital tight-binding model with bond potential $V_{p p \pi}$ $=-2.75 \mathrm{eV},{ }^{19}$ and a tight-binding model due to Charlier et $a l .{ }^{48}$ which incorporates the effects of both $s, p$ orbitals. Both models have been extensively tested, and deemed to give good results for the electronic properties of carbon nanotubes. The advantage of the single-band $\pi$-orbital model lies in its simplicity: because only $\pi$ orbitals are considered, it is possible to take advantage of the one-dimensional character of carbon nanotubes and study systems, whose size approaches that of realistic carbon nanotube devices. The Charlier model, on the other hand, is able to incorporate rehybridizations between the different $s, p$ orbitals and has a more realistic distance dependence. It is therefore ideal for the investigation of the electronic properties of the different defects. It is, however, considerably more costly. The approach that we have therefore taken is to investigate the properties with the $\pi$-orbital model and verify the results with the more realistic Charlier model. In general, there is very good agreement between the two models on all the general trends and universal features of the conductance. We note that unless otherwise stated, when displaying the results from the Charlier model, we have shifted the Fermi level of the tubes to coincide with $E=0$. Finally, it must be emphasized that all the structures whose electronic properties were investigated were obtained by carefully relaxing hightemperature, classical molecular dynamics configurations with a simulated annealing process in which the TersoffBrenner potential ${ }^{49}$ was used to model the carbon atoms.

To calculate shot noise, we make use of formulas developed via the Keldysh nonequilibrium Green's function formalism. ${ }^{41,42}$ The current fluctuations $\left\langle(\Delta I)^{2}\right\rangle$ over a frequency range $\Delta \omega$ are given by

$$
\left\langle(\Delta I)^{2}\right\rangle=\frac{2 e^{2} \Delta \omega}{h} \int_{E_{F}+e V_{L}}^{E_{F}+e V_{R}} d E \operatorname{Tr}[(1-T) T],
$$

assuming the low temperature limit. The other quantity of importance is the Fano factor $\gamma$ :

$$
\gamma=\frac{\left\langle(\Delta I)^{2}\right\rangle}{(2 e / h) \Delta \omega I},
$$

which is a measure of the deviation from the classical Poissonian behavior.

In order to simulate the effects of a magnetic field $\mathbf{B}$ oriented perpendicular to the nanotube axis, ${ }^{32-34}$ we use a vector potential $\mathbf{A}=(L B / 2 \pi) \sin [(2 \pi / L) x] \hat{\mathbf{y}}$, where the length $\mathrm{L}$ is defined in terms of the chiral vector $\mathbf{C}_{h}=n \mathbf{a}_{1}+m \mathbf{a}_{2}$ via $L=\sqrt{3} a_{C C} \sqrt{n^{2}+m^{2}+n m}$ with $a_{C C}=1.42 \AA$ and $\mathbf{a}_{1,2}$ the basis vectors. It has been shown that the effects of the external field can be recast in terms of a set of phase factors $\exp \left[(i e / \hbar c) \gamma_{i j}\right]$ which is introduced in the hopping integrals between sites $\mathbf{R}_{i}$ and $\mathbf{R}_{j}$. These may then be calculated with a Peierl's substitution: ${ }^{34}$

$$
\gamma_{i j}=\frac{2 \pi}{\phi_{o}} \int_{R_{i}}^{R_{j}} \mathbf{A} \cdot d \mathbf{r},
$$

where $\phi_{o}=h c / e$ is the flux quanta. Writing $\Delta x=X_{i}-X_{j}$ and $\Delta y=Y_{i}-Y_{j}$, simple algebra gives the following expressions for the phase factors:

$$
\gamma_{i j}(\Delta x \neq 0)=\left(\frac{L}{2 \pi}\right)^{2} \frac{\Delta y}{\Delta x} B\left[\cos \left(\frac{2 \pi x}{L}\right)-\cos \left(\frac{2 \pi(x+\Delta x)}{L}\right)\right]
$$

and

$$
\gamma_{i j}(\Delta x=0)=\left(\frac{L}{2 \pi}\right) \Delta y B\left[\sin \left(\frac{2 \pi x}{L}\right)\right] .
$$

In presenting the magnetic field effects, it is useful to recast the results in terms of a dimensionless parameter $\nu$ $=L /(2 \pi l)$, which is defined in terms of the inverse of the magnetic length $l=\sqrt{\hbar c / e B}$.

\section{RESULTS}

\section{A. Transmission through pristine nanotubes}

Before presenting our finite-sized nanotube data, we briefly review the dc transport of infinite carbon nanotubes, i.e., we shall refer to infinite nanotube devices as a nanotube coupled seemlessly to two other nanotubes of the same type. Metallic armchair nanotubes are characterized by the crossing of two bands at the Fermi level, so that the theoretical conductance, in units of $2 e^{2} / h \approx(12.9 k \Omega)^{-1}$ has a value of two and the conductance $G$ is a constant over this energy range. At higher (lower) energies, electrons are able to probe different subbands leading to an increase in $G$ that is proportional to the number of additional bands available for transport. The conductance curve therefore consists of a series of "down-and-up" steps, with step positions correlating with the subband edges that are marked by peaks in the local density of states (LDOS).

The behavior of $G$ for finite-sized $(10,10)$ armchair nanotubes coupled to the leads is shown in Fig. 2 for both the $\pi$-orbital and Charlier tight-binding models. The response of these tubes is strikingly different as $G$ now consists of a series of peaks where the nanotube has a transmissive level. As will be discussed, both the peak heights and positions are a reflection of the band structure of the nanotubes. To confirm the resonant behavior of the system, we have plotted both $G$ and the LDOS together. Since the latter measures electron dwell time inside the conducting nanotube, ${ }^{50,51}$ the LDOS is highly peaked whenever the energy of the tunneling electron matches that of a resonant state. Figure 2 shows that there is excellent correspondence between the conductance peaks and LDOS. Physically, this resonance behavior may be attributed to the scattering at the contact between the nanotubes and leads, which in our model is included in the self-energy terms of the Green's function. Such scattering contacts are, of course, absent in the infinite nanotubes previously analyzed. We note that experimentally, there is now considerably evidence for such resonant transmission. ${ }^{12-14}$ We also note that for the most part, the data presented here is for zero temperature. The effects of a nonzero temperature is essentially to broaden and "smear" out the resonant tunnel- 
ח-Orbital Model

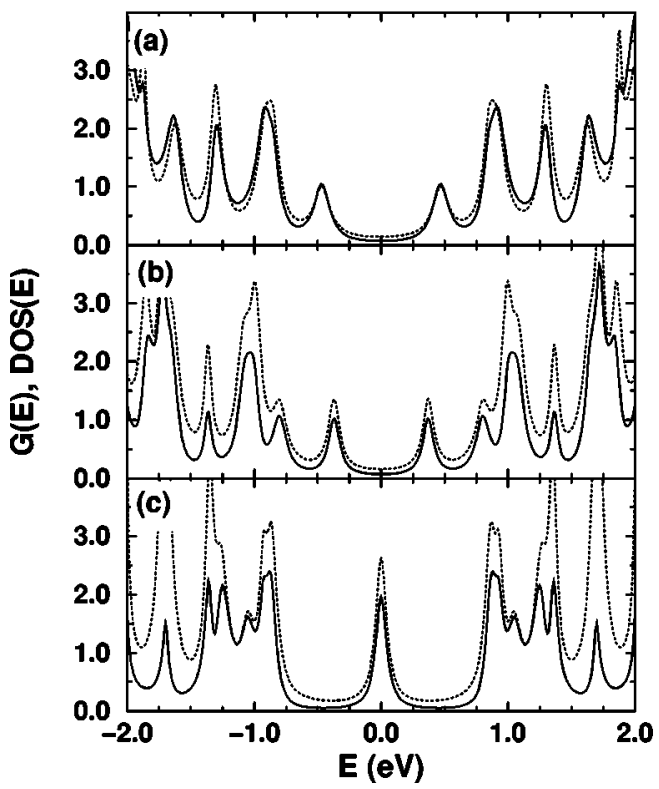

Charlier Model

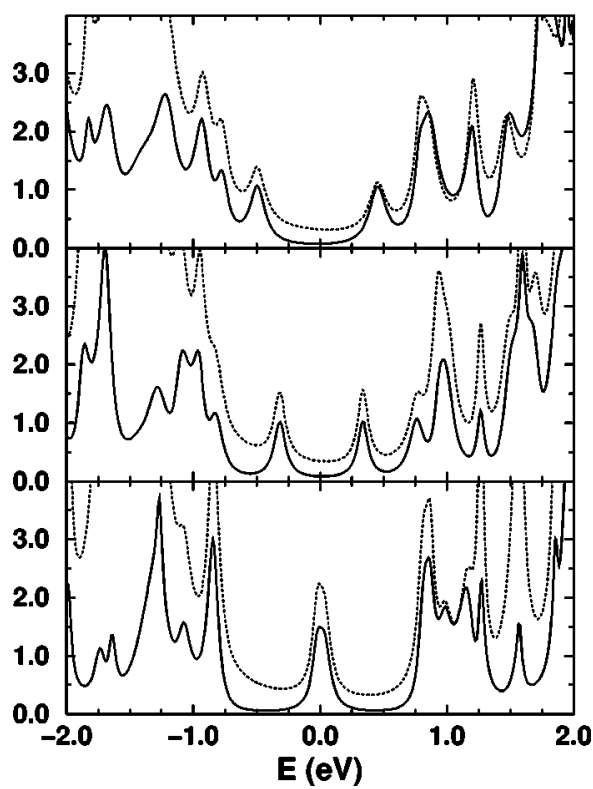

FIG. 2. Resonant tunneling peaks for finite-sized $(10,10)$ nanotubes as calculated with the $\pi$-orbital (left panels) and Charlier (right panels) models showing both off- and on-resonance devices. The solid lines mark the conductance spectra while the dotted lines denote the LDOS. Nanotubes with different number $(\mathrm{N})$ of unit cells are shown: (a) $N=6$; (b) $N=6$; and (c) $N=7$, and $\Gamma_{L, R}$ $=0.5$. ing peaks. Numerical investigations show that this effect is quite small for temperatures up to $300 \mathrm{~K}$, so that these effects can safely be ignored.

It is also evident from Fig. 2 that both positions and heights of the resonant peaks depend on the nanotube length. The off-resonant $(10,10)$ tubes consisting of 10 and 12 atomic layers, respectively (20 atoms per atomic layer), show a peak height of one conductance unit, while the onresonant tube of 14 atomic layers is characterized by a peak height of about two units. In fact, if one considers increasing the length of the armchair nanotubes layer-by-layer, then every third nanotube shows a peak at the Fermi level. Numeri- cally, we have checked this all the way out to 1000 atomic layers $(\approx 240 \mathrm{~nm})$. This result is also consistent with previous studies of the band gap for finite-length armchair nanotubes. ${ }^{52}$ These calculations showed that the gap in the eigenvalue spectrum oscillates between large and small values as tube length is increased, with the small-gap case corresponding to on-resonance systems. This is because for small values of the coupling $\Gamma$, the eigenvalues are close to the scattering states, which therefore show a similar behavior.

Physically, one can attribute both the peak height and periodicity of the on-resonance devices to the electronic

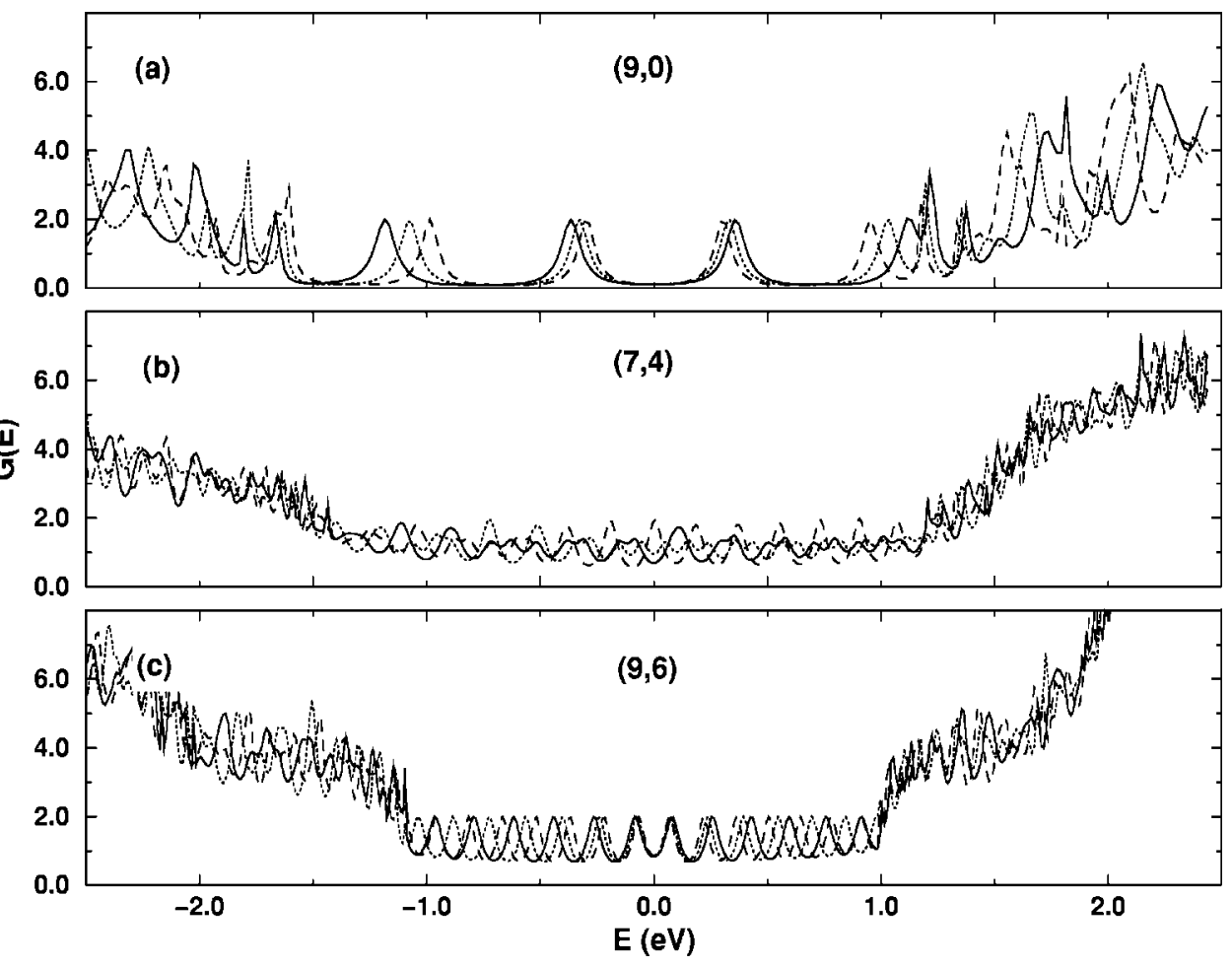

FIG. 3. Conductance spectra for nanotubes with different helicities as calculated with the Charlier model: (a) $(9,0)$ zigzag tubes (18 atoms per layer); (b) $(7,4)$ chiral (62 atoms per layer); and (c) $(9,6)$ chiral nanotubes $(114$ atoms per layer). The solid lines mark tubes consisting of $N=10$ unit cells; dotted line, $N=11$; and the dashed $N=12$, respectively, with $\Gamma_{L, R}=0.5$. 

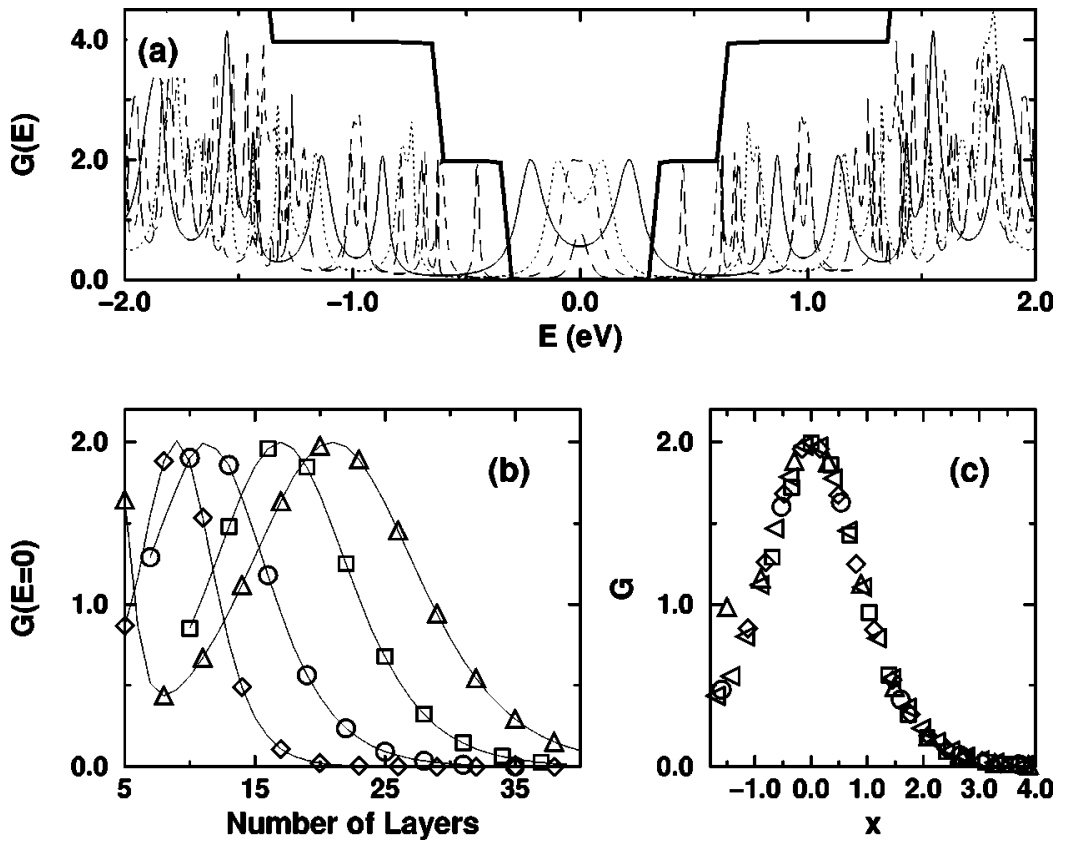

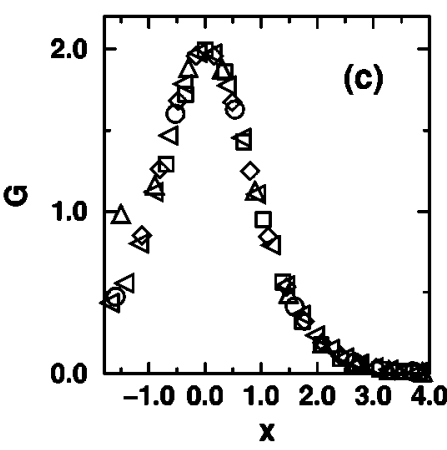

FIG. 4. Conductance spectra for short, finitesized semiconducting nanotubes, which display a resonant peak at the Fermi level that decays as the nanotube length increases. Here (a) shows the full spectra for $(17,0)$ nanotubes: dashed line, $N$ $=5$; dotted line, $N=10$; thin-solid line; $N=25$, long-dashed, $N=30$; thick solid line, infinte tube. In (b), we display the decay of the peak as a function of nanotube length for different semiconductor nanotubes: circles, $(10,0)$ tubes; squares, (10,5); diamonds, (14,0); triangles, $(17,0)$. As shown in (c), there is good scaling in the decay of this central peak $G^{*}$ as a function of the scaled nanotube length $x$, which is defined as $x=\left(N-N_{\max }\right) / N_{1 / 2}$, where $N_{\max }$ is the length where the maximum conductance is attained and $N_{1 / 2}$ is the length where the conductance peak height takes on half of its maximal value. For all runs, $\Gamma_{L, R}=0.5$. properties of the nanotubes. The band structure of armchair nanotubes consist of two non-degenerate bands that cross the Fermi level at $k=2 \pi / 3 a_{o}$, with lattice constant $a_{o}$. Finite tubes sample $k$ 's in units of $2 \pi / L$, where $L$ is the nanotube length. Clearly, only when $L=3 N a_{o}$ with integer $N$ can one probe the Fermi level exactly where the two bands overlap. Otherwise, for incommensurate lengths, one probes the other regions of the band structure that contain no overlap, ${ }^{53}$ and therefore give rise to peaks of height of unity. This then directly accounts for the peak pattern shown in Fig. 2.

Similar considerations account for the peak heights and distribution with respect to tube length for nanotubes with different symmetry, as illustrated in Fig. 3. For instance, the band structure of the $(n, 0)$ metallic zigzag tubes consists of two degenerate bands crossing the Fermi level at the $\Gamma$-point. Hence all the peaks will have a height of two. Since the $\Gamma$-point cannot be probed for tubes of finite length, we see no peaks at the Fermi level for the tubes as a function of length. As the tubes become longer, the peaks simply crowd in toward $E=0$.

The behavior of the metallic chiral tubes is qualitatively similar to either the armchair and/or zigzag tubes. The band structure of $(n, m)$ chiral tubes, for which the greatest common divisor $\operatorname{gcd}(n, m) \neq 3$, is qualitatively similar to that of the armchair tubes with two nondegenerate bands crossing at $k=2 \pi /(3 T)$, where $T$ is the length of a unit cell (typically an irrational number), so that one can expect the resonant tunneling peaks at the Fermi level to have a height of two and a repeat length of $3 T$. (For a detailed discussion of the nanotube band structure and unit cells, see Ref. 1.) Indeed, that is what we observe in the simulations except that in contrast to the armchair nanotubes, some of the other peaks

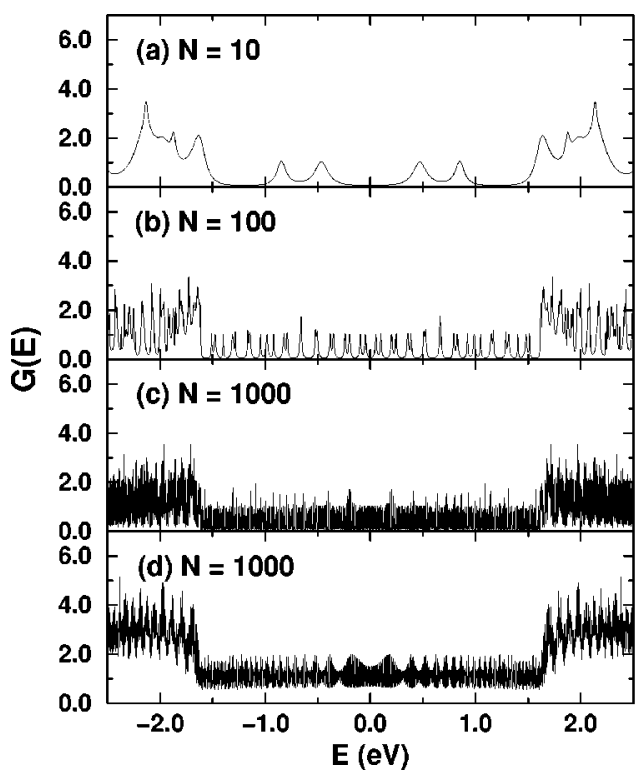

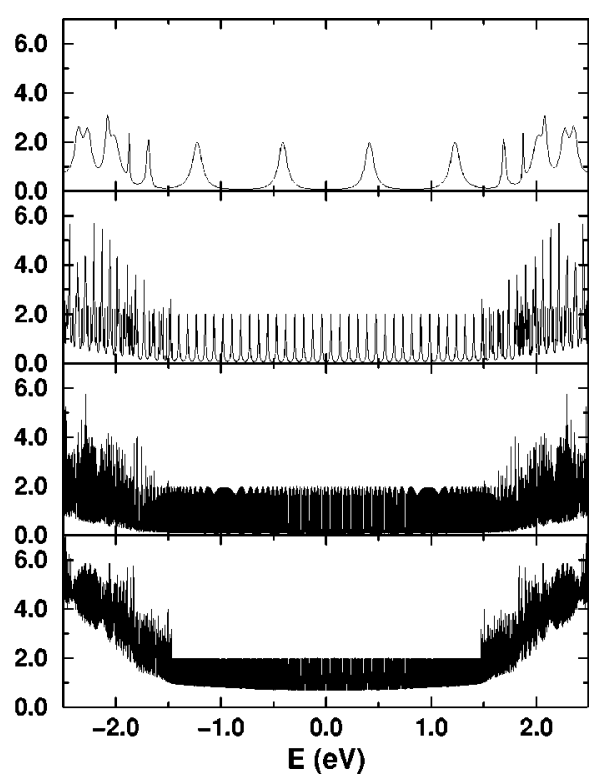

FIG. 5. Conductance spectra as the nanotube length becomes very large as calculated with the $\pi$-orbital model for the $(5,5)$ armchair nanotube (left panels) and the $(9,0)$ zigzag tubes (right panels). The nanotube lengths are (a) $N=10$; (b) $N=100$; and (c) and (d) are for $N=1000$, respectively. For (a)-(c), $\Gamma_{L, R}=0.5$ while $\Gamma_{L, R}$ $=2.0$ for $(\mathrm{d})$. As $\Gamma$ is increased, the peak widths become wider and begin to overlap. 
have a value that is between one and two. We attribute this difference to the proximity of the scattering states for this chiral nanotube which, when coupled to the leads which serves to broaden the peaks, overlap and merge to form peaks whose height varies between one and two units of conductance. The electronic properties of metallic chiral nanotubes for which $\operatorname{gcd}(n, m)=3$ are qualitatively similar to the metallic zigzag tubes, with two doubly-degenerate bands crossing at the $\Gamma$-point. And indeed, the conductance spectra for the $(9,6)$ system resembles that of the $(n, 0)$ tubes, as shown in Fig. 3.

We have also examined transmission through finite-sized semiconducting nanotube systems. Long, semiconducting nanotubes display the expected behavior of isolated peaks outside of the band gap. Surprisingly, we find that the very small nanotubes, consisting of less than about 40 atomic layers, are characterized by a central peak at the Fermi level which decays as the length of the nanotubes increases in a non-monotonic fashion. For instance, Fig. 4(a) shows a typical conductance signature for a $(17,0)$ nanotube, while Fig. 4(b) plots the decay of the central peak as a function of nanotube length for different semiconducting systems. The essential behavior consists of an initial increase in the height of the central peak at about $N \approx 5-15$ atomic layers, followed by a decay in the peak height. As shown in Fig. 4(c), there is scaling behavior in the falloff of the central peak height: by plotting the conductance $\mathrm{G}$ as a function of $x$ $=\left(N-N_{\max }\right) / N_{1 / 2}$, where $N_{\max }$ denotes the number of layers of the peak maximum and $N_{1 / 2}$ represents the number of layers where the conductance falls off to half its maximum value-i.e., it is a rough measure of the width or curvature of the central peak. We note that this display of metallic behavior for small systems is a characteristic of several semiconducting material systems. For instance, both linear carbon chains $^{54}$ and very narrow silicon wires display metallic behavior. ${ }^{55}$

Turning to the infinite limit, we note that down-and-up step structure must be recovered as the nanotube length becomes very large, irrespective of the nanotube helicity. We have investigated the approach to this limit, as shown in Fig. 5 , and find that all the nanotubes display the following general features. As the length increases, the number of resonant peaks in any given energy range increases and the spacing

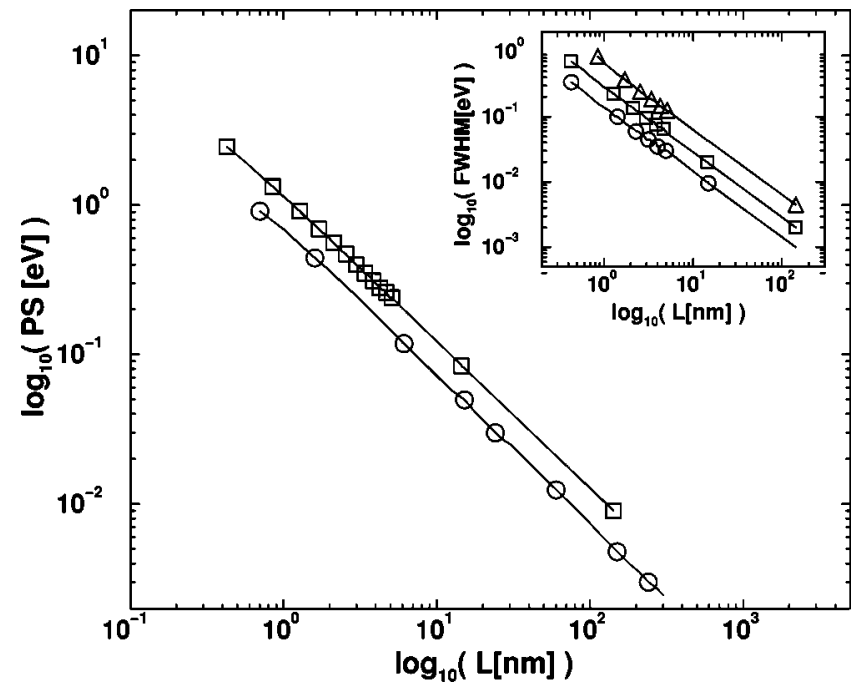

FIG. 6. Here we show some statistical characteristics of the resonant tunneling peaks. The main figure shows the spacing between peaks (PS) versus nanotube length $L$ in nanometers: circles, $(5,5)$ armchair nanotubes; diamonds, $(9,0)$ zigzag tubes. The inset shows typical, peak width at half maximum (FWHM) as a function of nanotube length for $(9,0)$ nanotubes for different $\Gamma$ 's: circles: $\Gamma_{L, R}=0.5$; squares, 1.0 ; and triangles, 2.0.

between any two given peaks decreases. At the same time, the width of these peaks decreases. These features are shown in Fig. 5 for both $(5,5)$ armchair and $(9,0)$ zigzag nanotubes. Because of the one-dimensional nature of the nanotubes, we have been able to probe nanotube lengths that are within a factor of 2 for some of the experimental systems, showing that the finite-size nature of the system persists at least out to hundreds of nanometers. Figure 6 shows some statistics for the spacing between peaks and the peak widths. Both of these fall off exponentially, which has important implications for the ac conductance, as we shall discuss later. ${ }^{56}$ Figures 5 and 6 also show the effects of varying $\Gamma$, which parametrizes the coupling to the leads. As $\Gamma$ increases, the peaks broaden and begin to overlap, so that the conductance spectra becomes continuous and constant. As best can be distinguished, changing $\Gamma$ has no effect on the number of peaks present, and otherwise simply shifts the peak width. It
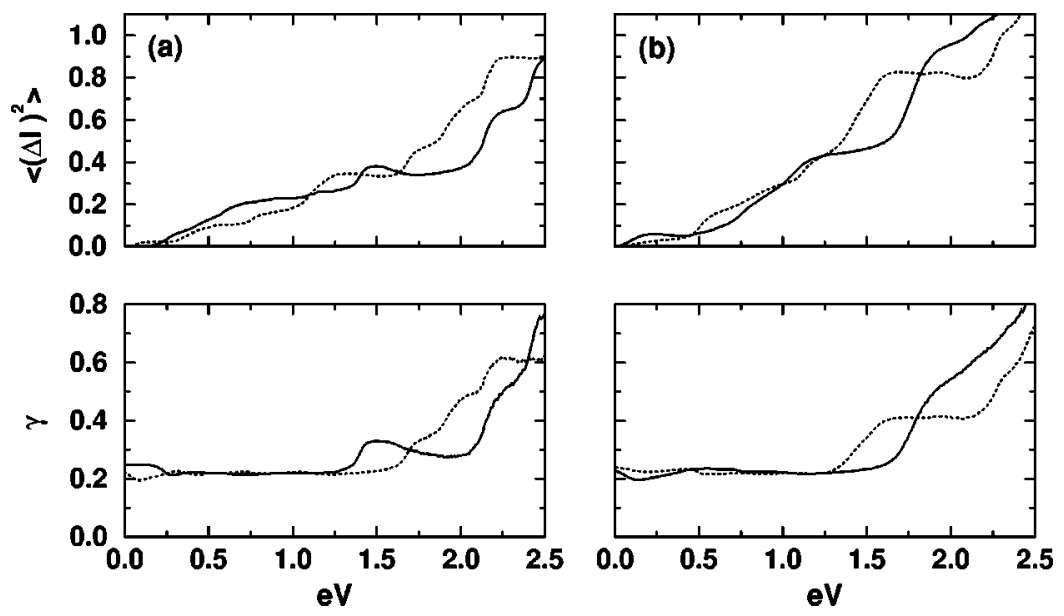

FIG. 7. Shot noise of carbon nanotube devices as obtained with the $\pi$-model. The top panel shows the variance of the current $\left\langle(\Delta I)^{2}\right\rangle$ as a function of electron voltage, while the lower panel shows the Fano factor $\gamma$. Here (a) is for $(5,5)$ tubes, and (b) for $(9,0)$ tubes; solid line$N=10$ layers; dotted line, $N=11$ layers, which represent on- and off-resonant devices for the respective nanotubes. 
a)

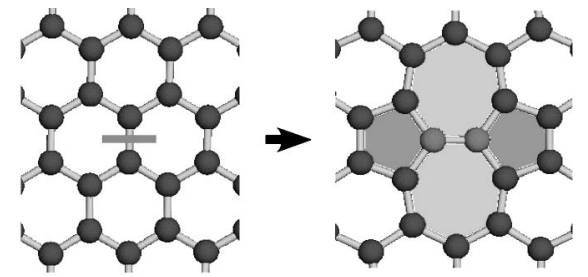

b)

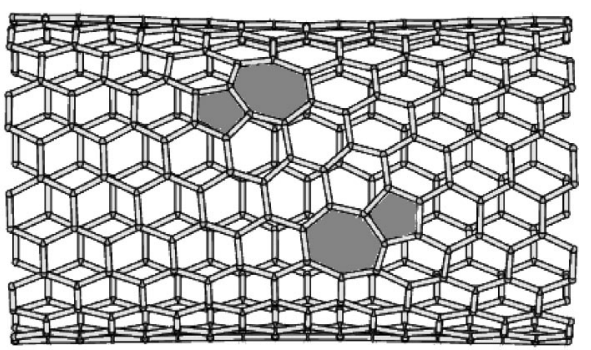

FIG. 8. Schematic of the (5-7-7-5) defect, which forms via the rotation of a $C-C$ bond in the wall of a strained nanotube (a) and (b). Upon annealing, the (5-7-7-5) defect may separate into two (5-7) pairs, as shown in (c).

has been shown ${ }^{57}$ that for a given energy level with wave function $\psi$, the coupling is estimated to be

$$
\Gamma \approx 2 \pi|\psi|^{2} C^{2} N_{o},
$$

where $C$ is the matrix elements between the atoms in the conductor and lead, and $N_{o}$ the metallic density of state per atoms at the Fermi level. The latter quantity, of course, varies significantly from atom to atom, so that one can expect the length that recovers the infinite limit to vary tremendously when different types of metals are used for the leads. For instance, $\mathrm{Na}, \mathrm{Mg}, \mathrm{Cu}, \mathrm{Ag}$, and $\mathrm{Au}$ all have total density of states that are less than 7.0 at the Fermi level. The systems that use these metals for leads should therefore display the resonant tunneling behavior over very much longer length scales than metals like $\mathrm{Mn}, \mathrm{Fe}, \mathrm{Pt}$, and $\mathrm{K}$ which are all characterized by relatively high densities of states. ${ }^{58}$

As a final consideration of the pristine nanotubes, we consider their shot noise, as given in Fig. 7. We have examined both on- and off-resonant devices for armchair tubes and tubes with a different helicity. In general, the same behavior is observed irrespective of the helicity of the nanotube. While the initial behavior of on-resonant devices is somewhat steeper, the two types of devices track each other relatively well. The basic statistics of the shot noise through the different nanotubes appears to be similar as evidenced by the Fano factor, which is mostly flat over a fairly large voltage region with the shot noise being suppressed by a factor of about $1 / 4$. This behavior is to be expected since, in the absence of any correlated scattering events, the connectivity is probably the major factor in determining the shot noise, which is the same for the different symmetry tubes. Note that in this calculation we assume a temperature regime, such that the many-body Coulomb interactions do not play a major role. At extremely low temperatures of a few degrees Kelvin,
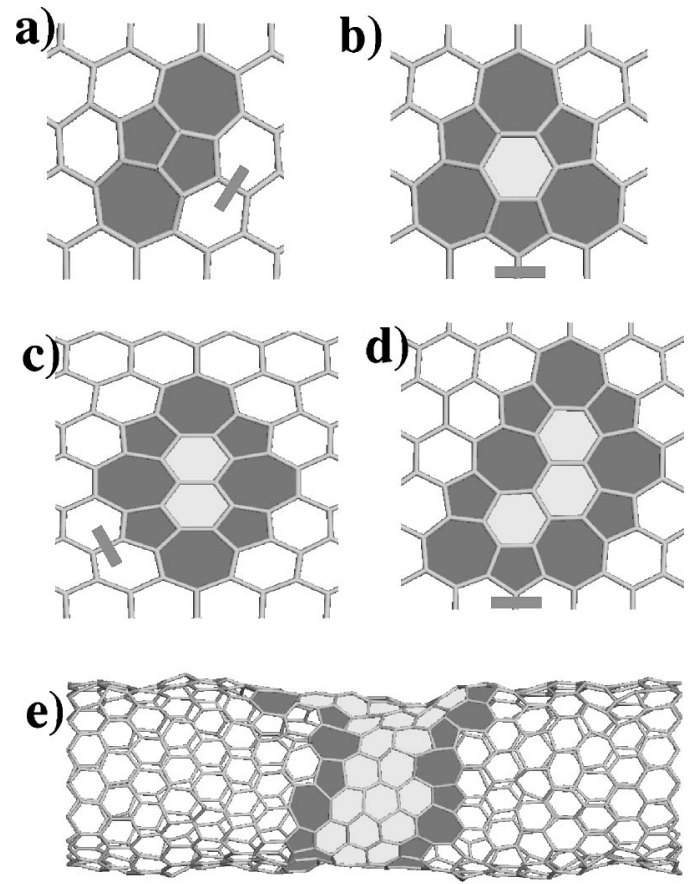

FIG. 9. Formation of extended defects via the incorporation of a $C_{2}$ dimer into the walls of a strained carbon nanotube: (a) the (75-5-7) defect; (b) rotation of bond emanating from one of the pentagons to form a defect with a single enclosed hexagon; (c) twoand (d) three-enclosed hexagons. Ultimately, the defect can wrap itself entirely about the circumference of a nanotube to form an electronic heterojunction, as shown for a $(17,0) /(8.8) /(17,0)$ quantum dot structure in (e).

it is likely that even further deviations from the classical shot noise value would appear because of possible Luttinger liquid effects.

\section{B. Transmission through defective nanotubes}

We have investigated the transmission through bent nanotubes and nanotubes with two sets of defects associated with strained nanotubes. What is probably the most common defect on nanotubes under tension is initiated via the rotation of a $C-C$ bond in the nanotube wall, i.e., a Stone-Wales transformation familiar from $C_{60}{ }^{45}$ that leads to the formation of a (5-7-7-5) defect, as illustrated in Fig. 8. The formation of this defect has been investigated with classical, tight-binding, and $a b$ initio simulations ${ }^{46}$ that show that it is energetically perferred on armchair nanotubes under tensions of 5\% or more. Once nucleated, this defect undergoes either ductile or brittle behavior depending on the temperature, rate of stress, and the helicity of the nanotube. Ductile or plastic behavior is typically associated with armchair nanotubes, and involves the separation of the (5-7-7-5) defect into two (5-7) pairs, which then glide about the circumference of the nanotube. Brittle behavior, on the other hand, is typically associated with formation of large rings that subsequently open to form a crack and ultimately lead to the breakage of the nanotube. Zigzag tubes tend to display brittle behavior, while chiral nanotubes are expected to show some degree of ductility. ${ }^{46}$ 

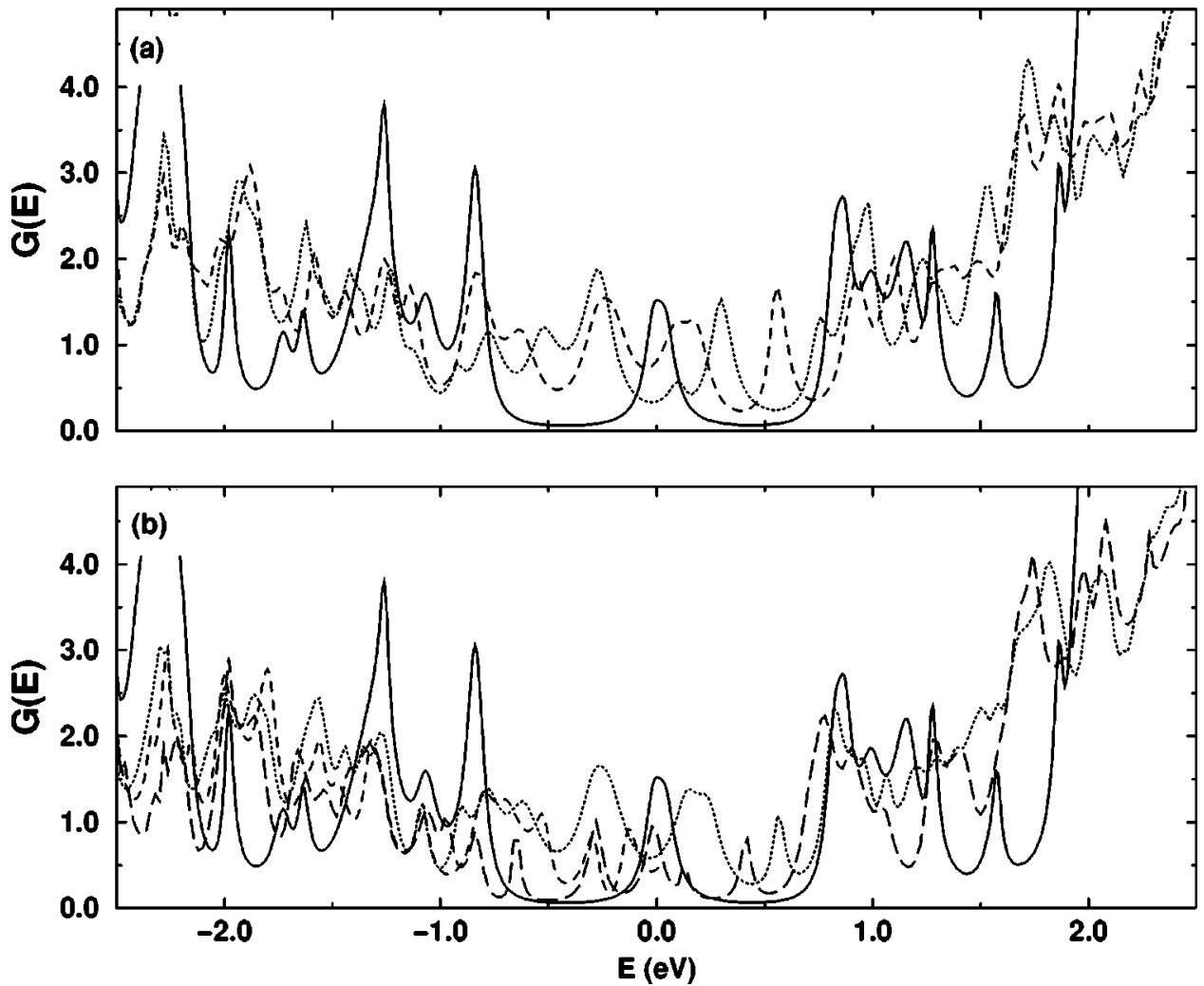

FIG. 10. Conductance signatures of defective $(10,10)$ nanotubes as obtained from the Charlier model. In (a) the solid line shows the pristine nanotube; the dotted line the (5-7-7-5) defect; and the dashed line the separated (5-7) pairs, as shown in Fig. 8(c). Similar results are shown in (b) for defects obtained via the incorporation of a $C_{2}$ dimer into a nanotube wall: solid linepristine nanotube; dotted line(7-5-5-7) defect; dashed linedefect with single hexagon; and the long dashed line-defect with two hexagons.
The second class of defects is associated with the incorporation of a $C_{2}$ dimer into the wall of a strained carbon nanotube. ${ }^{28}$ Here, the dimer initially forms a (7-5-5-7) defect, as shown in Fig. 9. Under 5-10\% strain, this defect undergoes substantial further evolution through a rotation of the bonds emanating from the vertex of the pentagons, thereby forming a series of rotated hexagons that are separated from the rest of the nanotube by a series of (5-7) pairs. This process continues and leads to the wrapping of the defect about the circumference of the nanotube, thereby forming a short segment of a tube with an altered helicity. This is interesting because, depending on the helicity of the initial
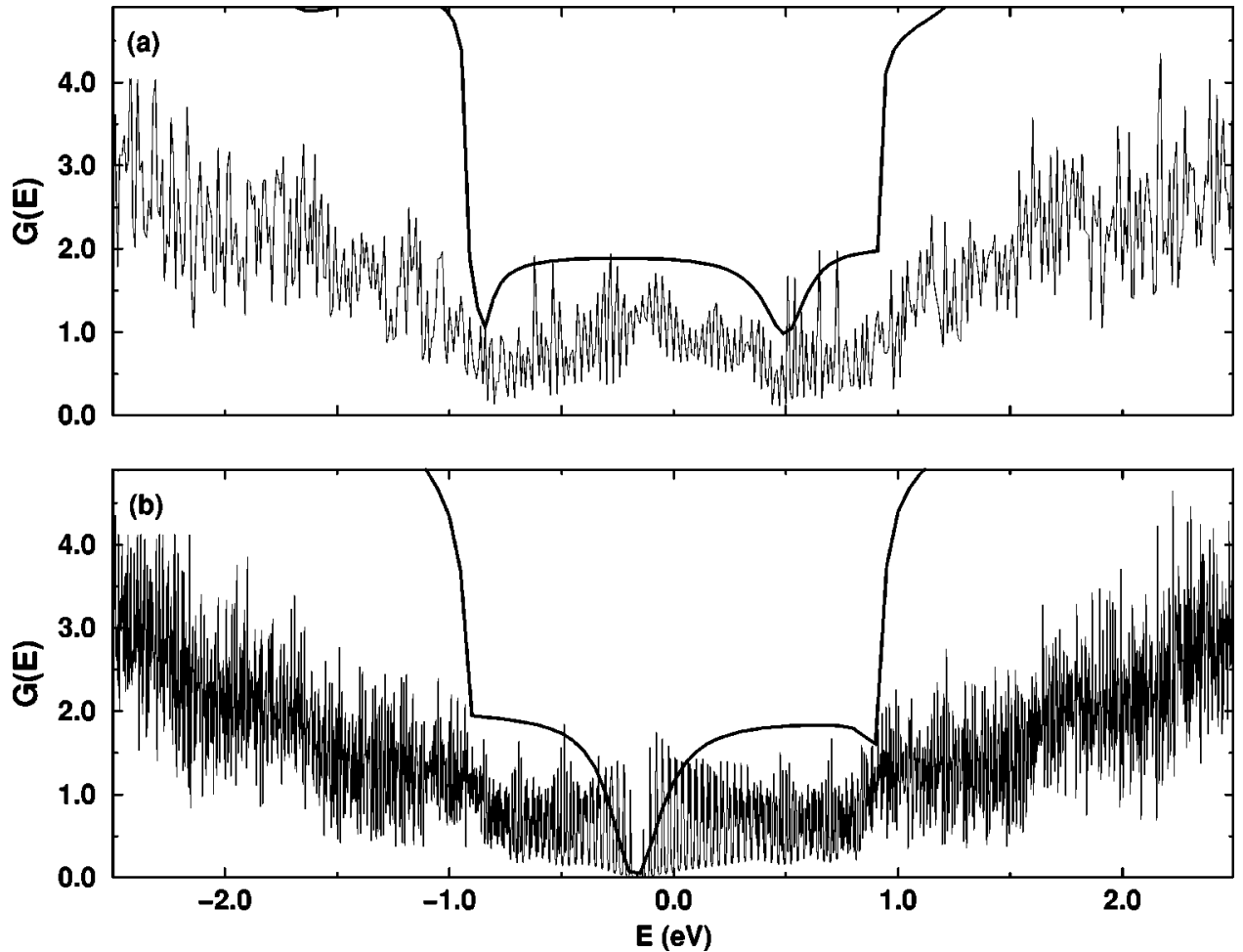

FIG. 11. Conductance signatures of very large, defective nanotubes as obtained with the $\Pi$-orbital model: (a) a $N=1000$ $(10,10)$ tube with a $(5-7-7-5)$ defect; (b) a $N=1000(10,10)$ tube with a single, rotated hexagon. The solid curve in both cases denotes the infinite limit. 
tubes, these defects naturally form different electronic heterojunctions. The formation of such structures is particularly favorable for the zigzag tubes, where the presence of addimers induces plastic transformations on what are otherwise brittle tubes. $^{28}$

Figure 10 illustrates the effect of these defects on the resonant transmission peaks for the $(10,10)$ tubes. In general, the effects of single defects of both classes leads to a reduction of the peak heights, both at the Fermi level and elsewhere. At the same time, the presence of defects can lead to appearance of new peaks that appear over a broad, nonzero background. For instance, for the case of the (5-7-7-5) defect there is an initial reduction at the peak height for $E=0$. At the same time, the conductance does not drop off to zero between the peaks but actually has values between $\sim 0.5-1.0$. As the tubes are annealed and the defect separates into two (5-7) pairs, there are further changes both in the peak height at the Fermi level and in the peak positions. Similar results are obtained for the (7-5-5-7) defect, although in this case, the initial reduction in the peak height is much more. However, as the defect evolves to incorporate more and more hexagons, the non-zero background disappears and a resonant peak structure emerges whose spectra is significantly different from that of the pristine nanotubes. Qualitatively similar results are observed as the length of nanotube becomes very large, as shown in Fig. 11. Ultimately, with larger values of the parameter $\Gamma$, the infinite limit is recovered which shows that individual defects only have a modest effect on the conductance properties. There is slight reduction at the Fermi level accompanied by more significant reduction through the development of "dips" out near the band edges.

As described previously, ${ }^{28}$ addimers on strained nanotubes yield extended defects that may ultimately form different electronic heterojunctions. As an example, we have examined transport through a quenched $(17,0) /(8,8) /(17,0)$ quantum dot structure and a $(8,8) /(17,0) /(8,8)$ structure that is a prototypical metal/insulator/metal (MIM) system. The former was formed by the annealing of addimers on a $(17,0)$ tube, while the latter was constructed by cutting the $(17,0) /$ $(8,8) /(17,0)$ structure and then properly repositioning the atoms to form the MIM. In contrast to the infinite case, both structures are characterized by a nonzero conductances, as shown in Fig. 12. For the quantum dot there is a small nonzero conductance for virtually all energy values about the

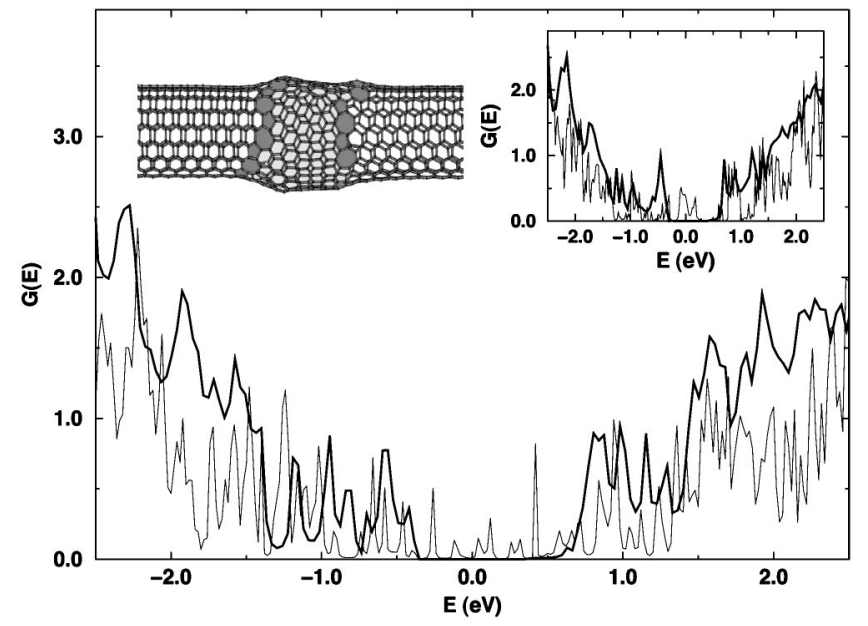

FIG. 12. Conduction signatures, as obtained with the $\Pi$-orbital model for a prototypical $(8,8) /(17,0) /(8,8) \mathrm{MIM}$ and a $(17,0) /(8,8) /$ $(17,0)$ quantum dot: thin solid line-finite-sized stuctures; thick, solid line-infinite limit.

Fermi level, while the MIM is characterized by sharp resonant peaks associated with the localized states of the structure. Other electronic heterojunctions were also examined, but for the most part, those results agree completely with that of the infinite case. For instance, the $(12,0) /(6,6)$ metal-metal interface is characterized by a large gap about the Fermi level, in agreement with previously published results. ${ }^{19}$

We have also examined the conductance through bent nanotubes of different helicities. The results, however, are very similar to those of the defective nanotubes already discussed. First, the structure of typical bent armchair and chiral tubes are shown in Fig. 13. For the most part, there are few structural changes in the tubes when the bending angle is small. And indeed, the conductance up to about $12^{\circ}$ displays only small differences from the unbent case. At larger angles of $24^{\circ}$ and higher, the differences are more substantial. There is a general reduction in the height of the peak at the Fermi level and a new conductance spectra emerges, as shown in Fig. 14. Ultimately, as the tubes become infinitely long, the bent nanotubes are driven towards opening gaps at the Fermi level, thereby inducing a metal/insulator transition.

\section{Effects of magnetic fields}

We now turn to the effects of externally imposed magnetic fields, restricting ourselves to the case of a field that is
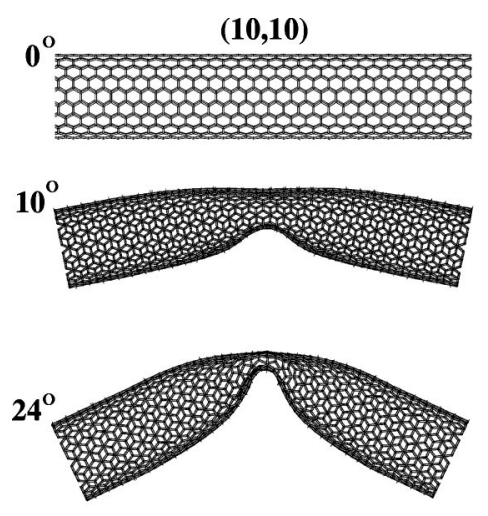

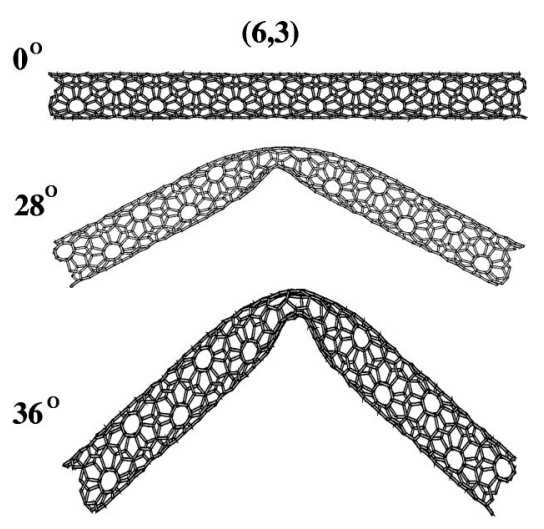

FIG. 13. Atomic structure of bent carbon nanotubes: left panels- $(10,10)$ tubes; right panels- $(6,3)$ nanotubes. From top to bottom, the bending angle is $0^{\circ}, 6^{\circ}, 24^{\circ}$, and $36^{\circ}$. 


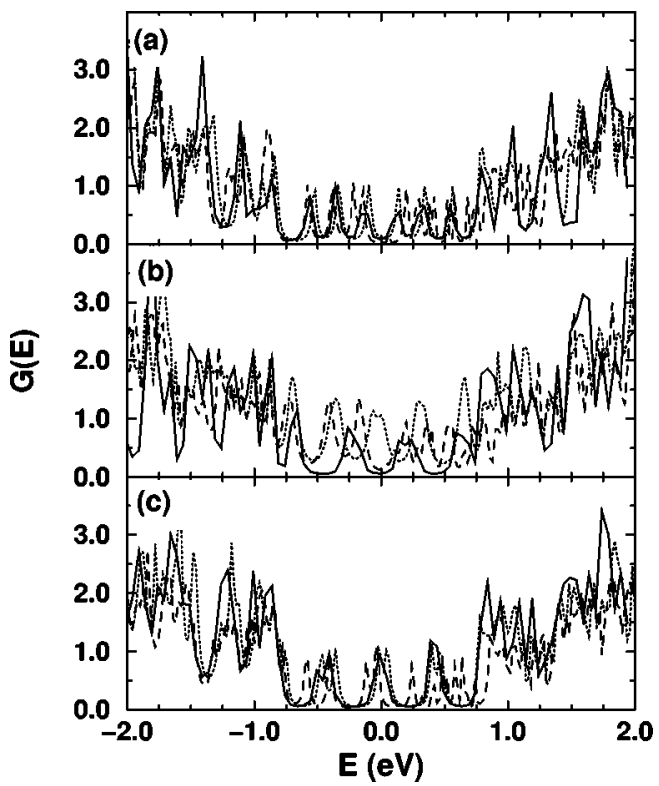

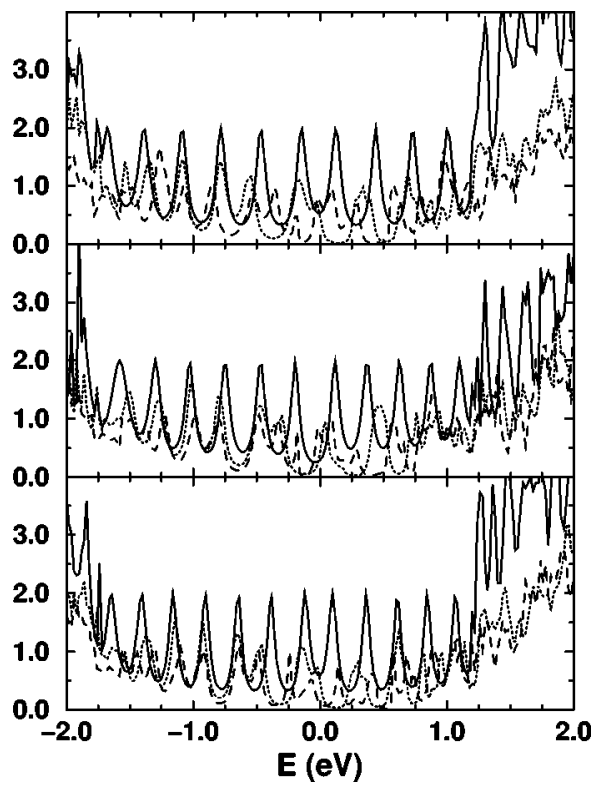

FIG. 14. Conductance spectra for bent nanotubes as obtained with the Charlier model: left panels- $(10,10)$ tubes; right panels- 6,3$)$ nanotubes. Here, the solid line denotes the unbent case $\left(0^{\circ}\right) ;$ dotted line- $-6^{\circ}$; dashed line $-24^{\circ}$ degrees for the left panel and $36^{\circ}$ for the right panel. The lengths of the tubes in atomic layers are (a) $N=30 ; N$ $=31$, and $N=32$, for the left panels and $N=5,6,7$ for (a) -(c) in the right panels, respectively. oriented perpendicular to the nanotube axis. The infinite limit has been explored in several publications, ${ }^{32-34}$ which show that the main effect of a $\mathbf{B}$ field is to induce a metal-insulator transition. Our results show similar features as a function of nanotube length. For instance, Fig. 15 shows conductance spectra for $(10,10)$ on- and off-resonance systems for different $\nu$. Clearly, the effect of the $\mathbf{B}$ field is to diminish (increase) the height of the peaks at the Fermi-level. Similar results are obtained for the zigzag tubes. Figure 16 plots the heights of conductance at the Fermi-level for tubes of different lengths, with the following generic results. For offresonance tubes with low-conductance at the Fermi level, increasing $\nu$ leads to a peak in the value of conductance for some nonzero value $\nu \sim 1.0$. By contrast, for the onresonance case at small values of $\nu$, the conductance at the Fermi level is nearly contant. At larger values of $\nu$, there is a marked decrease, followed by a more complicated peak structure. Thus the externally imposed $\mathbf{B}$ field can turn onand off-resonant devices into each other. These generic features are preserved as the nanotube length is increased. For instance, in Fig. 16(b) we have plotted the height of the conductance peaks at $E=0$ for nanotubes of length $N=5$ and $N=100$ unit cells. In both cases, the conductance is initially zero but then increases to form a peak structure as the parameter $\nu$ is increased. For larger sized nanotubes, this peak simply shifts inwards towards smaller values of $\nu$. Qualitatively similar results are obtained for tubes with different helicities.

\section{SUMMARY}

We have examined theoretically the dc quantum conductance through finite-sized carbon nanotubes coupled to featureless leads in the context of standard tight-binding models. Transmission through such systems takes place via resonant tunneling, which leads to a set of conductance peaks whose spectra depends strongly on the length and helicity of the nanotubes. In turn, this spectra is a reflection of the distribution of the scattering states of the nanotube systems, which in most cases strongly resembles the band structure of the infinite carbon nanotubes. It is also interesting that semiconducting nanotubes coupled to leads display metallic behavior when lengths less than $\approx 40$ atomic layers are considered.
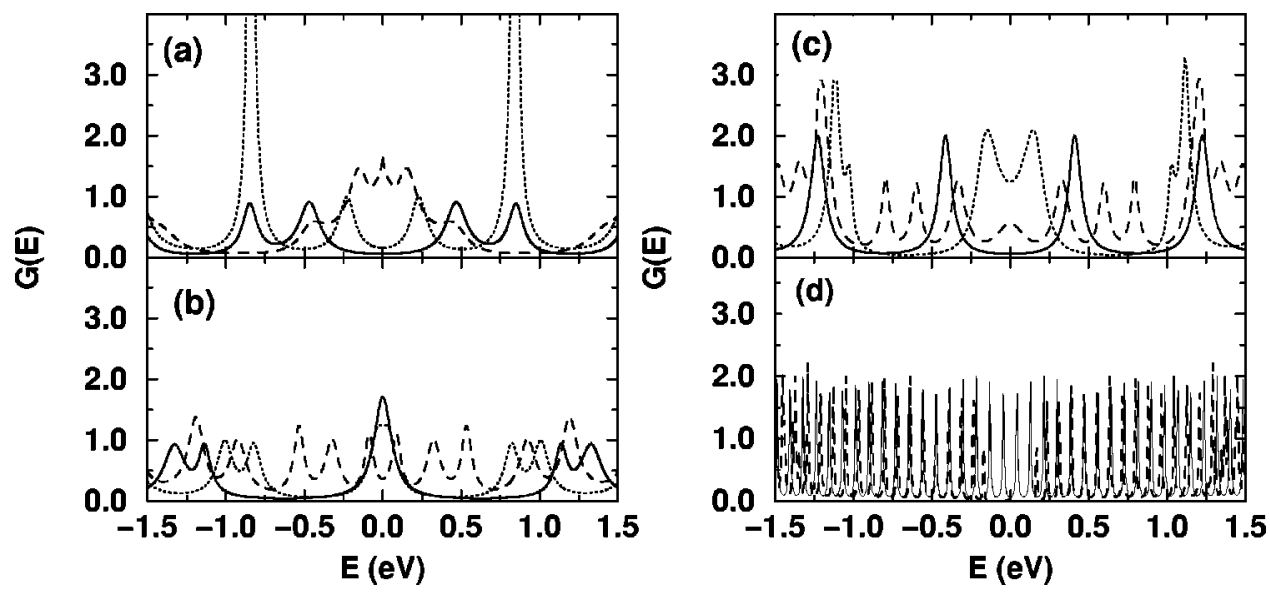

FIG. 15. Conductance spectra for $(5,5)$ armchair nanotubes (left panel) and the $(9,0)$ zigzag nanotubes (right panel) for different values of $\nu$ : solid line $-\nu=0$; dotted line $-\nu=0.75$; dashed line $-\nu=1.0$. Here for the armchair tubes (a) denotes the off-resonance $N=5$ and (b) the on-resonance $N=7$ case, respectively. For the zigzag tubes, (a) is for $N=5$ and (b) for $N$ $=100$. 

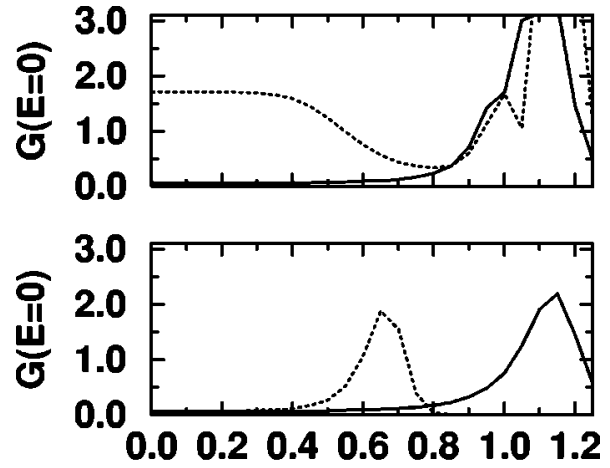

V

FIG. 16. Peak height at the Fermi level as a function of magnetic parameter $\nu$. Here (a) denotes results for the $(5,5)$ armchair tube with the solid-line marking the off-resonance case with $N=5$, and the dotted line the on-resonance case; (b) results for $(9,0)$ zigzag tubes with solid line for $N=5$ and the dotted line denoting $N$ $=100$, respectively.

As the length of the nanotubes becomes large, the transport behavior approaches that of infinite carbon nanotubes. However, the finite-size behavior persists out to lengths of hundreds of nanometers, which is the length scale most commonly probed in the current experiments. As nanotube length increases, the number of resonant tunneling peaks in a given energy interval increases. Both the distance between the peaks and peak widths decrease exponentially with length. Increasing the coupling between the leads and the nanotube broadens the peaks leading to overlap so that the infinite limit is approached at shorter lengths. This implies that metallic leads with a higher density of states at the Fermi level reach the infinite limit sooner.

We have also examined the conductance signatures of two different types of defects, plus that of bent carbon nanotubes. The first is based on the (5-7-7-5) defect, which forms via a bond rotation on strained nanotubes and is responsible for the initial mechanical response of nanotubes under tension.
The second set of defects is associated with the presence of addimers on strained nanotubes. This defect undergoes substantial further evolution, ultimately forming short segments of tubes of altered helicity separated from the main tube via (5-7) pairs. In general, the effect of the defects on the conductance signature is relatively modest. It consists largely of a decrease in the height of the conductance peaks at the Fermi level and a small shifting of the resonant tunneling peaks. As the nanotube length is increased, the resonant tunneling peaks merge to form dips in the conductance that lie either slightly above or below the Fermi level. Similar features are characteristic of bent nanotubes.

We have also examined the effects of externally imposed magnetic fields which are oriented perpendicular to the nanotubes. Generically, the effect of such fields is to decrease (increase) the height of the conductance peaks at the Fermi level depending on whether the nanotube device is on- or off-resonance. The acual value of the magnetic parameter where such a change takes place is, however, sensitive to length and helicity of the nanotube in the device.

In summary, we have examined the general features of the conductance spectra of finite-sized carbon nanotubes coupled to leads, along with the effects of defects and magnetic field effects. While there is good experimental evidence for many of the finite-size features explored here, all should be readily accessible and testable with careful experimentation. In closing, we note that we have also examined the ac conductance of both infinite ${ }^{30}$ and finite-sized nanotubes, ${ }^{56}$ which will be presented in a future publication.

We would like to thank Jerry Bernholc, Marco Buongiorno Nardelli, and Vincent Meunier for numerous scientific discussions. The following agencies are gratefully acknowledged for financial support: NSERC of Canada and FCAR of Quebec (H.G.); RGC grant (HKU 7115/98P) from the Hong Kong SAR (J.W.); and ONR N000014-98-0597 and NASA NAG8-1479 (C.R.). We also thank the North Carolina Supercomputing Center (NCSC) for extensive computer support.
${ }^{1}$ For reviews, see, for example, M. S. Dresselhaus, G. Dresselhaus, and P. C. Eklund, Science of Fullerenes and Carbon Nanotubes (Academic Press, San Diego, 1996); R. Saito, G. Dresselhaus, and M. S. Dresselhaus, Physical Properties of Carbon Nanotubes, ICP Press (World Scientific, Singapore, 1998); J. Bernholc, C. Roland, and B. I. Yakobson, Crit. Rev. Solid State Mater. Sci. 2, 706 (1997).

${ }^{2}$ S. Frank, P. Poncharail, Z. L. Wang, and W. A. de Heer, Science 280, 1744 (1998).

${ }^{3}$ J. W. G. Wildoeer, L. C. Venema, A. G. Rinzler, R. E. Smalley, and C. Dekker, Nature (London) 391, 59 (1998); J. W. Odom, J.-L. Huang, P. Kim, and C. M. Lieber, ibid. 391, 62 (1998).

${ }^{4}$ S. J. Tans, A. Verschueren, and C. Deeker, Nature (London) 393, 49 (1998).

${ }^{5}$ R. D. Antonov and A. T. Johnson, Phys. Rev. Lett. 83, 3274 (1999).

${ }^{6}$ R. Martel, T. Schmidt, H. R. Shea, T. Hertel, and Ph. Avouris,
Appl. Phys. Lett. 73, 2447 (1998).

${ }^{7}$ Z. Yao, H. Postma, L. Balents, and C. Deeker, Nature (London) 402, 273 (1999).

${ }^{8}$ S. Paulson, M. R. Falvo, N. Snider, A. Helser, T. Hudson, A. Seeger, R. M. Taylor, R. Superfine, and S. Washburn, Appl. Phys. Lett. 75, 2936 (1999).

${ }^{9}$ P. G. Collins, Z. Zettl, H. Bando, A. Thess, and R. S. Smalley, Science 278, 100 (1996); S. N. Song, X. K. Wang, R. P. Chang, and J. B. Ketterson, Phys. Rev. Lett. 72, 697 (1998); L. Langer, L. Stockman, J. P. Heremans, V. Bayot, C. H. Olk, C. Vana Haesendonck, Y. Bruynseraede, and J.-P. Issi, J. Mater. Res. 9, 927 (1994); L. Langer, V. Bayot, E. Grivei, J.-P. Issi, J. P. Heremans, C. H. Olk, L. Stockman, C. Van Haesendonck, and Y. Bruynseraede, Phys. Rev. Lett. 76, 479 (1996).

${ }^{10}$ A. Bezryadin, A. R. M. Verschueren, S. J. Tans, and C. Dekker, Phys. Rev. Lett. 80, 4036 (1998).

${ }^{11}$ D. H. Cobden, M. Bochkrath, P. L. McEuen, A. G. Rinzler, and 
R. E. Smalley, Phys. Rev. Lett. 81, 681 (1998).

${ }^{12}$ S. J. Tans, M. H. Devoret, H. Dai, A. Thess, R. E. Smalley, L. J. Geerligs, and C. Dekker, Nature (London) 386, 474 (1997).

${ }^{13}$ M. Brockrath, D. H. Cobden, P. McEuen, N. Chopra, A. Zettl, A. Thess, and R. E. Smalley, Science 275, 1922 (1997); M. Brockrath, D. H. Cobden, J. Lu, A. G. Rinzler, R. E. Smalley, L. Balents, and P. L. McEuen, Nature (London) 397, 598 (1999); A. Bachtold, C. Strunk, J. P. Salvetat, J.-M. Bonnard, L. Forro, T. Nussbaumer, and C. Schoenenberger, ibid. 397, 673 (1999).

${ }^{14}$ L. C. Venema, J. Wildoeer, J. Janssen, S. J. Tans, H. Temminck Tuinstra, L. Kouewenhovem, and C. Dekker, Science 283, 52 (1999).

${ }^{15}$ A. Bachtold, M. S. Fuhrer, S. Plyasunov, E. H. Anderson, A. Zettl, and P. McEuen, Phys. Rev. Lett. 84, 6082 (2000); M. Bockrath, J. Horne, A. Zettl, P. McEuen, A. G. Rinzler, and R. E. Smalley, Phys. Rev. B 61, R10 606 (2000).

${ }^{16}$ K. Tsukagoshi, B. W. Alpenaar, and H. Ago, Nature (London) 401, 572 (1999).

${ }^{17}$ Y. Zhang et al., Science 285, 1719 (1999).

${ }^{18}$ A. F. Morpurgo, J. Kong, C. M. Marcus, and H. Dai, Science 286, 263 (1999); A. Yu Kasumov et al., ibid. 284, 1508 (1999).

${ }^{19}$ X. Blase, L. X. Benedict, E. L. Shirley, and S. G. Louie, Phys. Rev. Lett. 72, 1878 (1994); Y. A. Krotov, D.-H. Lee, and S. G. Louie, ibid. 78, 4245 (1997); L. Chico, V. Crespi, L. X. Benedict, S. G. Louie, and M. L. Cohen, ibid. 76, 971 (1996); L. Chico, L. X. Benedict, S. G. Louie, and M. L. Cohen, Phys. Rev. B 54, 2600 (1996); V. H. Crespi, M. L. Cohen, and A. Rubio, Phys. Rev. Lett. 79, 2093 (1998); P. McEuen, M. Bockrath, D. Cobden, Y. G. Yoon, and S. G. Louie, ibid. 83, 5098 (1999); H. J. Choi, J. Ihm, S. G. Louie, and M. L. Cohen, ibid. 84, 2917 (2000).

${ }^{20}$ H. J. Choi, J. Ihm, Y. G. Yoon, and S. G. Louie, Phys. Rev. B 60, R14 009 (1999).

${ }^{21}$ L. Chico, M. P. L. Sancho, and M. C. Munoz, Phys. Rev. Lett. 81, 1278 (1998).

${ }^{22}$ W. Tian and S. Datta, Phys. Rev. B 49, 5097 (1994).

${ }^{23}$ R. Tamura and M. Tsukada, Phys. Rev. B 55, 4991 (1997); 58, 4882 (1998).

${ }^{24}$ M. P. Anantram and T. R. Govindan, Phys. Rev. B 58, 4882 (1998).

${ }^{25}$ A. A. Farajian, K. Esfarjani, and Y. Kawazoe, Phys. Rev. Lett. 82, 5084 (1998).

${ }^{26}$ M. Buongiorno Nardelli, Phys. Rev. B 60, 7828 (1999); M. Buongiorno Nardelli and J. Bernholc, ibid. 60, R16 338 (1999).

${ }^{27}$ A. Rochefort, Ph. Avouris, F. Lesage, and D. Salahub, Phys. Rev. B 60, 13824 (1999); A. Rochefort, D. Salahub, and Ph. Avouris, Chem. Phys. Lett. 297, 45 (1998).

${ }^{28}$ D. Orlikowski, M. Buongiorno Nardelli, J. Bernholc, and C. Roland, Phys. Rev. Lett. 83, 4132 (1999); Phys. Rev. B 61, 14194 (2000).

${ }^{29}$ H. Mehrez, J. Taylor, H. Guo, J. Wang, and C. Roland, Phys. Rev. Lett. 84, 2682 (2000).

${ }^{30}$ C. Roland, M. Buongiorno Nardelli, J. Wang, and H. Guo, Phys. Rev. Lett. 84, 2921 (2000).
${ }^{31}$ Y. Wei, J. Wang, H. Guo, H. Mehrez, and C. Roland (unpublished).

${ }^{32}$ R. Saito, G. Dresselhaus, and M. S. Dresselhaus, Phys. Rev. B 50, 14698 (1994).

${ }^{33}$ H. Ajiki and T. Ando, J. Phys. Soc. Jpn. 62, 1255 (1993); 62, 2470 (1993).

${ }^{34}$ S. Roche and R. Saito, Phys. Rev. B 59, 5242 (1999).

${ }^{35} \mathrm{~J}$. M. de Jong and C. W. J. Beenakker, in Mesoscopic Electron Transport, Vol. 345 of NATO Advanced Studies Institute Service E, edited by L. L. Sohn, L. P. Kouwenhoven, and G. Schoen (Kluwer, Dortrecht, 1997).

${ }^{36}$ M. Buettiker, J. Math. Phys. 37, 4793 (1996).

${ }^{37}$ M. Henry, S. Oberholzer, C. Struck, T. Heinzel, K. Ensseln, H. Holland, and C. Schoenenberger, Science 284, 296 (1999); W. D. Oliver, J. Kim, R. C. Liu, and Y. Yamanoto, ibid. 284, 299 (1999).

${ }^{38}$ G. Iannaccone, G. Lombardi, M. Macucci, and B. Pelligrini, Phys. Rev. Lett. 80, 1054 (1998).

${ }^{39}$ O. M. Bulashenk, J. M. Rubi, and V. A. Kochelap, Phys. Rev. B 61, 5511 (2000).

${ }^{40}$ A. Rekleitis and L. Reggiani, Phys. Rev. B 60, 11683 (1999).

${ }^{41}$ M. Buettiker, Phys. Rev. Lett. 65, 2901 (1990).

${ }^{42}$ Y. Wei, B. Wang, J. Wang, and H. Guo, Phys. Rev. B 60, 16900 (1999).

${ }^{43}$ R. Landauer, Nature (London) 392, 658 (1998).

${ }^{44}$ T. Gramespacher and M. Buettiker, Phys. Rev. Lett. 81, 2763 (1998).

${ }^{45}$ A. J. Stone and D. J. Wales, Chem. Phys. Lett. 128, 501 (1986).

${ }^{46}$ M. Buongiorno Nardelli, B. I. Yakobson, and J. Bernholc, Phys. Rev. B 57, R4 277 (1998); Phys. Rev. Lett. 81, 4656 (1998).

${ }^{47}$ S. Datta, Electronic Transport in Mesoscopic Systems (Cambridge University Press, Cambridge, 1995).

48 J.-C. Charlier, Ph. Lambin, and T. W. Ebbesen, Phys. Rev. B 54, R8 377 (1996).

${ }^{49}$ J. Tersoff, Phys. Rev. Lett. 61, 2879 (1988); Phys. Rev. B 37, 6991 (1988); D. Brenner, ibid. 42, 9458 (1990).

${ }^{50}$ M. Buettiker, Phys. Rev. B 27, 6178 (1983).

${ }^{51}$ J. Wang, Y. J. Wang, and H. Guo, Appl. Phys. Lett. 65, 1793 (1994).

${ }^{52}$ A. Rochefort, D. R. Salahub, and Ph. Avouris, J. Phys. Chem. B 103, 641 (1999).

${ }^{53}$ V. Meunier, P. Senet, and Ph. Lambin, Phys. Rev. B 60, 7792 (1999).

${ }^{54}$ N. D. Lang and Ph. Avouris, Phys. Rev. Lett. 81, 3515 (1998); 84, 358 (2000).

${ }^{55}$ J.-L. Mozos, C. C. Wan, G. Taraschi, J. Wang, and H. Guo, Phys. Rev. B 56, R4351 (1997).

${ }^{56}$ D. Orlikowski, J. Wang, H. Guo, and C. Roland (unpublished).

${ }^{57}$ W. Tian, S. Datta, S. Hong, R. Reifenberger, J. Henderson, and C. Kubiak, J. Chem. Phys. 109, 2874 (1998).

${ }^{58}$ D. A. Papaconstantopoulos, The Band Structure of Elemental Solids (Plenum Press, New York, 1986). 